\title{
Remembrances of Gilbert
}

Gilbert Baumslag was one of the leading infinite group theorists of the second half of the twentieth century. Through his own work, his students, and his mentoring, he had a profound effect on both the direction and interests in group theory. There is an ongoing seminar on his work given at Cornell University and various talks from this seminar can be found on the internet. The year he organized on combinatorial group theory at MSRI in 1989 ushered in much of the interest on automatic groups and hyperbolic groups. We note the paper by Alonso, Gersten, Shapiro, and Short explaining Gromov's ideas on hyperbolic groups and which came out of that conference as one of foundational works in geometric group theory. Gilbert's work and influence has been extremely wide, from classical combinatorial group theory, involving embedding theorems, and theory of nilpotent and one-relator groups to automatic and hyperbolic groups; the development of algebraic geometry over group with Myasnikov and Remeslennikov, which was instrumental in the proof of the Tarski theorems; the use of the variety of group representations with Peter Shalen; the development of groupbased cryptography. In addition to all this work in theoretical group theory, there was also his work on the Magnus project and all that it entailed in terms of computer implementations of group theoretic procedures. Since group theoretic procedures do not necessarily terminate, the Magnus project work as well as the work on group-based cryptography, veered greatly into computer science applications.

\section{Ben Fine and Gerhard Rosenberger}

Ben Fine began working with Gilbert through the Group Theory seminar as soon as I completed my own thesis work under Wilhelm Magnus. Gilbert was extremely helpful and always willing to listen to work and to give ideas. After the Friday seminar talk and either before or during dinner, a great many ideas for theorems and proofs flowed freely - most coming from Gilbert. He was fiercely protective of his own work as any great mathematician should be, but he was an extremely generous colleague who was willing to help anyone who needed it. Up until his death, he had an active group working with him consisting of both his own students and others. Gerhard Rosenberger and Ben Fine began working with Gilbert in the mid 1980s when some his work on the variety of group representations with Peter Shalen and our work with Jim Howie on essential representations overlapped. While Ben worked in the CAISS lab, lunchtime conversations with Gilbert led to a great deal more work particularly in group-based cryptography which got to Gerhard via email. We especially like material we did on provable password security. He became our co-author, along with Martin Kreuzer, on our book "A Course in Mathematical Cryptography," published by DeGruyter in 2015.

In 2000, I (Ben Fine) mentioned to Gilbert that the Magnus framework would be ideal with statistical evaluations, where the problems of whether the algorithms ter- 
minated or not did not appear. In Gilbert's inimitable style, he said "great idea. You go ahead and do it.” That began a 15-year project at CAISS developing CAISS-Stat that involved the programming work of Xiaowei Xu, Yeagor Brjukhov, P. C. Wong, and others as well as the work of Gilbert and myself. We developed what I felt was an excellent package which if further developed properly would have rivaled SAS and SPSS. What we developed up to a point was excellent, but we learned just how difficult it is to remove bugs from a multiuser software package. We also learned how difficult it is to actually market a new idea - no matter how good it is. I also learned how enthusiastic Gilbert could become. We ran several very nice and instructive seminars on CAISS-Stat. Through all of this work, it was more that just mathematics. We had conversations about all conceivable topics (we agreed on all things political). I considered Gilbert my good friend and mentor and I miss him.

\section{Chuck Miller}

Looking us up individually on MathSciNet, one finds a graphic showing that we were each others most frequent collaborator. We each had a number of other collaborators, and Gilbert was a lot more productive than I was. But we had a productive and enjoyable collaboration over many years. Our collaboration began with a mistaken example. In about 1974, I was a junior faculty member at Princeton, and Gilbert had returned to CUNY the year before. We saw each other fairly often in the NY Group Theory Seminar. One day he phoned me to tell me about a group that he and Frank Cannonito had constructed because he knew I would be interested.

Their group was (supposedly) locally free but could not be imbedded in a finitely presented group. He described the construction, but I could not immediately see how it worked. After a day or so thinking about this, I came up with a proof that every locally free group could be imbedded in a finitely presented group. So their purported example could not exist. It was also clear the method applied in more general circumstances where all the isomorphisms between finitely generated subgroups can be recursively enumerated. One simply realizes all of these isomorphisms in a large HNN extension which is then embeddable in a finitely presented group. After a few phone calls and mail messages, Gilbert was convinced. At his suggestion, we joined forces and eventually published four papers on this and related topics. Years earlier, in 1963-1964, I was a masters degree student in a subject on finitely presented groups that Gilbert taught at NYU.

There were several appealing aspects of Gilbert's subject. Some tools used were free groups, amalgamated free products, and HNN extensions. Basic questions were: when are groups finitely presented or finitely generated and the same for subgroups? One learned these constructions can be used to make new groups with interesting properties; for instance, the non-hopfian Baumslag-Solitar group BS $(2,3)$ they had recently concocted. Also one studied decision problems and showed the Adian-Rabin theorem that most properties are not recognizable. I found the connections with logic 
and the ability to construct things of particular interest. After that year, I moved to Illinois which had strengths in algebra and logic, and eventually Bill Boone was my $\mathrm{PhD}$ supervisor.

So Gilbert and that one subject had a strong influence on my mathematical interests and career. In 1976, I moved to Melbourne, but managed to visit the US fairly regularly. In the fall of 1979, I was on sabbatical resident at IAS when we began another collaborative effort on the homology of finitely presented groups with Eldon Dyer. We showed that any suitably given sequence of abelian groups could be realized as the homology sequence of a finitely presented group. Although this is a remarkable result, it is frustratingly short of a characterization. Like all the collaborations I have been involved in, this had elements of social enjoyment as well as mathematical pleasure.

Over the next 10 years, I was heavily involved in administration in Melbourne and visits to New York were less frequent. When I did came to New York, Gilbert often had a project in mind that he hoped we might do something toward. Resulting from one such project was our short paper "Some odd finitely presented groups" which we later jokingly called our most underrated paper. In it, we constructed a nontrivial finitely presented group which maps onto its direct square. So it is non-hopfian. A related group has a finitely generated derived group which is isomorphic to its direct square.

Starting in 1991, Gilbert became quite involved in the project to develop the Magnus computational group theory package. During much of that year, I was in New York and was also involved. Among other things, I wrote a collection of programs for manipulating words and subgroups in free groups. I also began collaborations with Gilbert and Hamish Short and eventually Martin Bridson.

After 2000, my obligations in Melbourne lessened and I visited New York more regularly. Gilbert was still concerned with the Magnus project and funding, but he managed time for lots of small research projects. He had a talent for suggesting projects one could make progress on in finite time. The results were generally interesting if not all that deep. He was also very generous with help for students and colleagues. For one such project, we were trying to better understand a group constructed by B. H. Neumann in the 1930s. But we only talked about this group while riding on the crosstown bus. We could just pick up where we had left off on a previous trip. This was good sport. With all the mathematical jargon, we were sure other passengers thought we were very strange.

Gilbert was a good friend and a very talented mathematician. We enjoyed each others company and doing mathematics together. He was remarkably generous with ideas and particularly helpful to students.

There is another aspect of his personality that I admired. When dealing with someone at say a counter in an office or a teller or an official, he would chat with them in an understanding way that indicated appreciation of their circumstance. It often put them at ease and made their lives seem a bit brighter, as though he was spreading a little joy. 


\section{Martin Bridson}

Gilbert Baumslag was my friend. We shared passions - for cricket, for arguing about how the world should be, for a measure of carousing, and above all for mathematics, especially the mathematics of group theory, about which he taught me a very great deal. For many years, starting in 1989, it was through conversation that he nurtured my appreciation of the elegance and achievements of pre-geometric group theory. But his great influence on our subject is in large part a function of his ability to express his many insights with such clarity in his writing. He was a master of explaining his solution to hard problems by exhibiting concise examples, and he had a well-honed skill for illuminating key ideas without stinting on rigor.

It is remarkable to reflect on how often Gilbert's papers opened doors to new worlds that held many wonders. One such paper that has been of particular significance in my life is Gilbert's article with Jim Roseblade on the subgroups of a direct product of two non-abelian free groups. Gilbert and Jim showed that, on the one hand, the array of finitely generated subgroups is strikingly diverse, while on the other hand, the only finitely presented subgroups are the obvious ones. This insight, and the homological techniques used to establish it, were the first key steps in what became a remarkably rich theory of subdirect products of hyperbolic and related groups, a subject that was the scene of many adventures for Gilbert, myself, Jim Howie, Chuck Miller, and Hamish Short (in various combinations) over 15 years.

Gilbert and I built many groups together but only published four papers. This is an inadequate indication of how much his mathematics influenced mine. A more accurate picture emerges if one scans the list of references in my other papers: he is there in many of them, for a variety of reasons - sometimes for his work on isoperimetric inequalities, subdirect products or equations over groups, but also for his seminal work on metabelian and nilpotent groups, profinite completions, and non-hopfian groups. His insights have populated my universe with great characters that I would not otherwise have known. My son, James, is a gifted cricketer in whom Gilbert took a particular delight. After Gilbert died, I pinned an email from Gilbert next to a picture on my office wall showing James in the act of bowling. I had shared that picture with Gilbert and his typically brief reply was one of the last things he wrote to me: "I envy you James. Love to you both, Gilbert.”

"We are all completely mad," he would often say. But oh what a life-affirming madness it was. I miss him greatly.

Martin Bridson, Oxford, January 2019

\section{Anthony Gaglione and Dennis Splellman}

Our Reflections on Gilbert Baumslag:

Dennis recalls attending a talk of Paul Schupp in which he was discussing groups with a certain property - call it P. (Dennis does not remember what P was!) Schupp's 
theorem was that finitely presented (or maybe finitely generated?) groups are generically - P. He justified the importance of his theorem with the assertion that unless you are Gilbert Baumslag, concrete examples of such groups are notoriously difficult to find. Such was the respect for Gilbert's power among his contemporaries. Not only does Gilbert's prolific outpouring of significant research merit recognition but also his encouragement of others.

Dennis also recalls the late Seymour Lipschutz relating to him Seymour's discussing a result of his with Gilbert and Gilbert replying that the result implies a solution to the conjugacy problem for cyclically-pinched one relator groups and that Seymour must publish. Thus, also in his encouragement of others, Gilbert helped advance the frontiers of our science.

In the New York Group Theory Seminar, Magnus had posed the question of whether or not the surface groups are residually finite. In a paper published in 1962, Gilbert established the stronger result that the surface groups are residually free. Here, he introduced the technique of big powers. For this, we are forever in his debt as we use that very technique over and over again in our own work. Implicit in Gilbert's 1962 paper is the fact that the surface groups are not only residually free but even freely discriminated in the sense that one can distinguish finitely many distinct elements by mapping into a free group. This paper along with a paper published in 1967 by his brother Benjamin was the basis for our result (independently proven by Remeslennikov) that, among finitely presented non-abelian groups, the freely discriminated ones are precisely the universally free examples. Incidentally, having mentioned the New York Group Theory Seminar, we would be remiss in not pointing out that that seminar's storied longevity is in no small measure due to Gilbert's stewardship for many years. Consulting Hanna Neumann's classic monograph on "Varieties of Groups," we see that Gilbert along with Bernhard, Hanna and Peter M. Neumann introduced (what we shall now call) varietally discriminating groups. Given a nontrivial variety of groups $V$, a group $G$ in $V$ is said to discriminate $V$ provided given finitely many words $w\left(x_{1}, \ldots, x_{n}\right)$ such that none of the equations $w\left(x_{1}, \ldots, x_{n}\right)=1$ is a law in $V$, there are elements $g_{1}, \ldots, g_{n}$ in $G$ such that simultaneously $w\left(g_{1}, \ldots g_{n}\right)=1$ for all the $W$. A group $G$ is then varietally discriminating provided it discriminates the variety it generates. Suppose $c$ and $r$ are integers with $c>2$ and let $N$ be free of rank $r$ in the variety $N_{c}$ of groups nilpotent of class at most $c$. Then, since $N$ is universally equivalent to the free group of countably infinite rank in $N_{C}, N$ discriminates $N_{c}$. From that, it follows that $N$ is varietally discriminating. Below are listed three papers in which we had collaborated with Gilbert (and for which we are indebted for his clear prose.) We want here to discuss one of these only; namely, the one on the nondiscrimination of nilpotent groups (see [3]). That would appear to contradict our discussion of free nilpotent groups above but here Gilbert was instrumental in promoting a different concept of discriminating group.

(It is somewhat amusing that Gilbert confessed to having forgotten his decades earlier definition joint with the Neumanns!) The newer definition of discriminating 
group is equivalent to the following: A group is discriminating provided it discriminates its direct square. From that, one immediately deduces that a discriminating group is universally equivalent to its direct square. With Gilbert's guidance, using the technique of Malcev completions (at which Gilbert was a master), we proved that no nilpotent group is discriminating unless it is abelian. (Torsion-free abelian groups are easily proven to be discriminating.) An ad hoc argument, without using the above theorem, that the groups $N$ alluded to earlier cannot be discriminating in the newer sense follows. The fact that the centralizer of any element outside the $(c+1)$-st term of the upper central series is abelian is captured by a universal sentence which is true in $N$ but false in $N \times N$. The theorem on nondiscrimination of nilpotent groups was proven independently using centralizer dimensions by Myasnikov et al. Although we have for decades studied the intersection of group theory and model theory, we both started our careers in the commutator calculus. Tony recalls that on a visit to the U.S. Naval Academy, Gilbert suggested to us that we study that ranks of the lower central quotients of free polynilpotent groups. This study ultimately led to the publication of a paper in which we gave recurrence relations to effectively compute the ranks of the lower central quotients of any free abelian by nilpotent group.

\section{References}

[1] G. Baumslag, O. Bogopolski, B. Fine, A. M. Gaglione, G. Rosenberger, and D. Spellman, On some finiteness properties infinite groups, Algebra Colloquium, Vol. 15 (2008), 1-22.

[2] G. Baumslag, B. Fine, A. M. Gaglione, and D. Spellman, Reflections on discriminating groups, Journal of Group Theory, Vol.10, (2007), 87-99.

[3] G. Baumslag, B. Fine, A. M. Gaglione, and D. Spellman, A note on the nondiscrimination of nilpotent groups and Malcev completions, Combinatorial Group Theory, Discrete Groups, and Number theory, AMS Contemporary Mat. Series Vol. 421, (2006), 29-34.

\section{Doug Troeger}

CAISS was founded to bring together the Science and Engineering communities at City College for the sharing of gains in mathematics, computer science, and related disciplines, and exploring the practical extensions of these gains. Gilbert Baumslag was the founding director of CAISS.

Building on the software package MAGNUS developed under Baumslag's leadership in the 1990s, the primary goals were to apply algebra and group theory to a wide array of applications, and to extend and expand the approach to computer algebra embodied in MAGNUS.

CAISS' ties to computer science were formalized with Baumslag's transfer to the Department of Computer Science in 2007. He was the key to recruiting N. Fazio, R. Gennaro, L. Gurvits, and W.Skeith to join the computer science faculty, with simultaneous 
appointment to CAISS. CAISS remains a vital center at City College; R. Gennaro currently serves as director. Projects illustrating Baumslag's role in bridging computer science and mathematics initiated by CAISSin the years 2000-2011 included efforts to:

1. Combine Axiom, MetaPrl, and MAGNUS to create software joining theorem proving and symbolic computation (A Next-Generation Computational Science Platform, proposal to NSF ITR, with S. Artemov, G. Baumslag, S. Cleary, T. Daly, J. Hickey, A. Nogin, W. Sit, and D. Troeger [2003]);

2. Port MAGNUS to the Sharp SL5600, a Linux-based PDA (G. Baumslag, T. Daly, J. Niu);

3. Use finitely presented groups as a replacement for social security numbers, for access control in data bases and electronic communications, leveraging computations carried out on hand-held devices running MAGNUS (Social Security Numbers, Electronic Communication and Finitely Presented Groups, proposal to NSF ITR, with G. Baumslag, S. Cleary, T. Daly, A. Kawaguchi, C. Miller [2003]; Universal Passwords and Cryptography Based on Group Theory, proposal to NSF ITR, with G. Baumslag, S. Cleary, T. Daly, A. Kawaguchi, and M. Anshel [2004]);

4. Extending AXIOM to allow indefinite computing in arbitrary mathematical domains (Parametric Computations in Axiom: Towards Indefinite Symbolic Computing, proposal to NSF CCF, with G. Baumslag, S. Cleary, T. Daly, W. Sit, and D. Troeger [funded 2006-2008]);

5. Enhance MAGNUS to (a) take full advantage of parallel computation on multiple CPUs - specifically on CAISS' 132-node Beowulf cluster, (b) integrate with the general purpose computer algebra system SAGE, (c) exploit functional programming to provide computational tools for working with infinitely presented groups, and (d) support a new interface for visual programming (Interactive Computation with Finitely Presented Groups, proposal to NSF, with G. Baumslag, P. Brinkman, Y. Bryukhov, C. Miller, and D. Troeger [2008]);

6. Use the MAGNUS front-end as a zero learning curve interface to a variety of backends, such as GAP, a linear algebra package, and a statistics package, resulting in CAISS-Stat (G. Baumslag, Y. Bryukhov, T. Daly, M. Dean, B. Fine, X. Xu, and M. Zyman);

7. Use finitely presented groups as a source of computer games, leading to Expacon (G. Baumslag, Y. Bruykhov);

8. Use the group randomizer system from MAGNUS for challenge-response security (Challenge Response Password Security Using Combinatorial Group Theory, G. Baumslag, B. Fine, Y. Bryukhov, and D. Troeger, Groups - Complexity - Cryptology, Volume 2, Issue 1, pp. 67-81 [2010]);

9. Use noncommutative groups for homomorphic encryption (Provable Security from Group Theory and Applications, proposal to NSF TC, N. Fazio, W. Skeith, V. Shpilrain, and G. Baumslag [funded 2011-2016]). 
CAISS contributed to the bridging of the mathematics and computer science departments as well by organizing a number of conferences and workshops of interest to both and held on the City College campus. These included Software for the Working Mathematician [2003], Axiom Conference [2005], Computation and Complexity [2006], Visualization Day [2008], Security and Privacy Day [2011], and Faces of Modern Cryptography [2011]. In addition, CAISS maintained a small Linux laboratory for the use of undergraduates, facilitating their participation in many of the Center's projects. T. Daly and R. Bruykhov offered courses on open-source programming using this lab, which was also used to host summer research programs for talented high school students.

\section{The Gilbert Group}

Definition 0.1. The Baumslag Group: advisees and protegees of Gilbert Baumslag during his last few decades; Kati Bencsath, Mariana Bonanome, Anthony Clement, Peggy Dean, Sal Liriano, Steve Majewicz, Gretchen Ostheimer, Marcos Zyman

\section{Beginnings}

Peggy: I first met Gilbert when I was an undergraduate and he was my Abstract Algebra professor in 1975. What joy that class was! Gilbert led us into wonderland and let us explore. I also took non-Euclidean geometry with Gilbert, and again he guided us through strange and marvelous places. Gilbert became my unofficial advisor at that time, and it was he who explained that I must go to graduate school, and helped me make it happen.

Gretchen: Gilbert was a mentor to me. My advisor took me to meet him in 1993 to get feedback about a problem we had identified as a possible thesis. Gilbert said it was a hard problem, maybe too hard. And then, when I solved it and started writing it up, I discovered that Gilbert himself had solved it many years prior! We joked that that's one liability of having such a huge body of work it was impossible for him to remember even his own results! It all worked out well: I was able to generalize the result and to begin my career as an independent researcher.

Steve: Every Friday, the Graduate Center offers various seminars. One of them was the Group Theory Seminar. I think it was in 1998 that I attended my first seminar. I remember being one of several students who would join the professors for coffee and cookies before the seminar (you can guess who some of the other students were - the Baumslag Group). I was not very sociable at the time, so I didn't interact with anyone. One person in the crowd with an accent that I was unfamiliar with attracted a lot of attention, including my own Gilbert. He was very comical and intelligent, and seemed to be the kind of person that I would like to learn from. I did not know if he taught at the GC, nor did I know his teaching style. Luckily for me, he was the speaker at the seminar. He 
gave a fascinating and intriguing talk in the area of combinatorial group theory. I immediately became interested in learning more about the subject, and I wanted Gilbert to teach me.

Anthony: I met Gilbert Baumslag between 2000 and 2001, when I took a group theory course he was teaching. I was working toward becoming an algebraic topologist. After my first class with Gilbert, I immediately changed my mind. Gilbert's teaching was smooth and captivating. He made group theory not only accessible, but even somewhat easy for me to follow. His style of joking throughout his lectures succeeded in making his audience feel completely relaxed and engaged as we learned.

Marianna: In 2001, I took a combinatorial group theory course at the CUNY Graduate Center and was fortunate to have Gilbert as my professor. What a magical time! I was finding my way as a graduate student and walking into Gilbert's class was like coming home.

Marcos: I am forever honored and humbled to have crossed paths with Gilbert Baumslag. In 2001, I audited a course he gave on combinatorial group theory at the CUNY Graduate Center. I had never seen such remarkable teaching in my life. It was because of him that I became a PhD student. Ever since that initial encounter, Gilbert's teaching and advice marked me in a profound way, both mathematically and personally. I never stopped attending his lectures from then on.

\section{Teaching}

Marianna: Gilbert was dynamic, supportive and full of humor in the classroom. He wrangled his students into group theoretic explorations requiring patience, skill, and creativity. He made every topic new at each revisiting and we his students happily revisited with him. He made me understand that it is okay to pull apart an idea, a theorem, a proof, a construction, again and again for weeks and years at a time. For years, I continued to take every class I could with Gilbert, even after graduating, sometimes taking the same class several times. I still return to my notes from these classes. They are prized possessions. Gilbert's spirit lives on in all of our teaching styles. The Baumslag Group, strives to inject joy, awe, and appreciation of mathematics in our classrooms; it is our way to honor our dear friend.

Peggy: Twenty years later, having left graduate school for a job, the time was right to go back and finish my degree. Gilbert was now on the CUNY GC faculty. I started taking his topics in group theory course, every semester for about 4 semesters. About midway through each lecture, Gilbert would ask us his signature question: Are you feeling weak, or are you feeling strong?” After my course work was complete, I kept sitting in on his courses along with other members of the Baumslag Group. There were often more unregistered students than registered!

Marcos: Gilbert was the embodiment of generosity. He loved discussing and sharing ideas with everyone. In the spring of 2007, Gilbert taught a course in finitely generated 
solvable groups at the CUNY Graduate Center. He gave his notes to some members of the Baumslag Group to organize them, fill in details, produce examples, expand them, and add our own thoughts. This is how I came to learn about semidirect products, wreath products, HNN-extensions, and, of course, finitely generated solvable groups. The notes eventually became a book. This is an example of who Gilbert was. He was all about offering projects, stimulating ideas, and teaching wonderful mathematics. He did all this because he enjoyed it, with boundless energy, and a remarkable sense of humor.

Anthony: Following that first class with Gilbert, I started reading some of his papers, even though I could not fully understand most of them. I met with Gilbert several times afterwards, and he directed me to read a particular paper, "On generalised free products.” This was the beginning of Gilbert becoming my unofficial advisor. A year or two later, I was still unsure whether he was my official advisor. I did not wish to disrupt the momentum we had established; we had been men at work. One afternoon when Gilbert was speaking with one of his colleagues I overheard him saying, "my student Anthony"; then I knew I was officially Gilbert's student.

Steve: Gilbert had a way of explaining difficult subject matter that was understandable for all of the students. He made the course enjoyable by throwing a few jokes around here and there. And he would always ask the audience if they had questions. One day, I met with Gilbert after class to ask for guidance. At that time, I was interested in nilpotent groups and exponential A-groups. Little did I know that Gilbert wrote a book on nilpotent groups. This was certainly meant to be! Gilbert took me under his wing and gave me a thesis project.

\section{Doing Mathematics}

Anthony: Gilbert Baumslag was a very kind, generous, and loving teacher and advisor. I met with Gilbert, religiously, every week for about 5 years. During my last year of meeting with Gilbert, I was having some difficulty completing my $\mathrm{PhD}$ thesis problem. He consoled and encouraged me by saying "There are lots of people out there with PhDs in Mathematics. I know that you know much more than many of them." That statement, coming from Gilbert, bolstered my self-confidence. Gilbert then left for Europe for 3 months. During his absence, I worked with renewed vigor and inspiration. Just before Gilbert returned, I solved and completed the problem. Gilbert will always have a special place in my heart. I am extremely grateful to have spent some time on this earth with him.

Marcos: Interacting with Gilbert was electric. His sheer passion for mathematics and for teaching sustained me throughout graduate school and sustains me still. I often remember those Friday evenings, on our way to dinner after the Group Theory seminar, walking around Manhattan with Gilbert and other seminar goers. Gilbert would discuss math in the street, over dinner, everywhere; always motivated by deep intellectual curiosity. His presence is still felt. 
Peggy: Gilbert became my advisor for my PhD thesis. It took me forever. Gilbert continues to be my inspiration for mathematics to this very day. Because of Gilbert, Marianna and I joined forces to write a book on "A Sampling of Remarkable Groups." When I lay awake at night struggling with some problem, I always thought, "What would Gilbert do?"

Marianna: I had the privilege of working as a research assistant for Gilbert at his lab at CCNY for several years and he was also my thesis advisor for a year. He had a knack for knowing exactly what challenge to pose to me. I recall him writing problems on the board in his office (playing with elements from free products with amalgamation) for me to solve. He would leave me to struggle, chat with someone else, then come back to check on me, always with his white ceramic mug in hand (never a disposable cup in sight!) Gilbert generously doled out ideas, matching them to interest and ability. When I told him I had an idea to use methods from quantum computation to solve decision problems in combinatorial group theory, he encouraged me to find a physicist to advise and guide me, which I did. Gilbert continued to guide me mathematically and pushed me to innovate. Peggy and I still argue: "What would Gilbert do?" What we don’t argue about anymore is "He must have made a mistake here!”

Steve: Friday was the best day of the week, the day that I would go to the GC for a class taught by Gilbert, go to a seminar and sit with Gilbert, and/or discuss group theory with Gilbert. He was a person that you just wanted to hang out with. I can still hear him say "Every finitely generated torsion-free nilpotent group has a poly-infinite cyclic and central series. Make sure that you can prove this in your sleep.” After completing my $\mathrm{PhD}$, I had the idea of writing a book on nilpotent groups, written for both students and professors in the field. Gilbert gave me some ideas. He emphasized that it would take a lot of work and a long time to complete. He advised me that no matter how good the book is, there will always be critics. I pursued the project with Anthony and Marcos. During the years that we worked on it, Gilbert helped us fill in some of the missing pieces from the literature. His support, encouragement, and guidance was truly heartening, especially at times when the work became tiresome and endless. The book, entitled 'The Theory of Nilpotent Groups,' was finally published in 2017. Unfortunately, Gilbert passed on before seeing it. It is dedicated to him. I often think about Gilbert and how he has touched the lives of so many people. God bless him.

Gretchen: In 2005, I started working with Gilbert and Chuck Miller on a question about metabelian groups, and then we just kept on working. Eventually, we published a paper about intersections of subgroups and then we moved our attention to decompositions of nilpotent groups. We were trying to figure out under what conditions it could be decided whether a given nilpotent group was decomposable. My last meeting with Gilbert and Chuck together was in August 2014. We were able to finally see our way to an algorithm for deciding decomposability in the case of finitely generated torsion-free nilpotent groups. Gilbert got his diagnosis that month, and was gone within 2 months. It turned out there were quite a few details yet to be worked out, and the work was fi- 
nally published in 2016. Gilbert was a friend. We didn't talk about personal stuff that much. But Gilbert knew when I was going through difficult personal stuff. He got it, he just got it that life is hard sometimes and that's the way it is, and knowing that Gilbert got it was always a great solace to me. I really don't know how I got so lucky to have been able to work with Gilbert for almost 20 years. It is certainly one of the things in this life for which I feel most grateful. Maybe it wasn't luck, but rather Gilbert's incredible generosity. He gave to so many of us, regardless of our academic stature, that which was most precious: his treasure trove of questions, his time, and his serious attention.

\section{Addendum}

Peggy: My current husband, David, attended my defense. He knew nothing of mathematics, and at one point Gilbert looked over at him and asked "David, are you understanding any of this?" Gretchen, who was sitting next to David, said "He's been taking notes this whole time!" Here are the results of those notes (to be sung to the tune of “Old McDonald”):

\section{The Group Theory Group Anthem}

Gilbert Baumslag had a group non-Abelian.

And this group was torsion-free, torsion, torsion-free.

Reprise:

Torsion here, and free free there,

A torsion portion, and it's free,

We are on a modding spree.

Gilbert Baumslag had a group non-Abelian.

And this group was nilpotent, nil, nil pot-pot-tent.

With a nil-nil here and potent there,

Nil-a-nil and pot-pot-tent,

An isomorph? Ah there it went.

Reprise

And this group was para-free, para-para free,

With para here and free-free there,

Para free isn't really free,

It's a fact, o can't you see?

With a nil-nil here and potent there,

Reprise

And this group has an Ore Domain, Ore Ore Domain.

With an Ore here and Domain there,

Ore Ore, Do-Do main,

This Ore Domain is one big pain.

With para here and free-free there,

$A+B$ equals sub-prime $P$ 
With a nil-nil here and potent there,

Reprise

And this group has a skew fields, skew-skew, skew-skew field.

With a skew-skew here and a field-field there.

Here a skew, there a field,

This skew fields is no big deal.

With an Ore here and Domain there,

With para here and free-free there, $\mathrm{B}+\mathrm{B}$ equals sub-prime $\mathrm{P}$

With a nil-nil here and potent there,

Reprise

And then there is a wreath product. Wreath-wreath, prod-prod-duct.

With wreath-wreath here and a product there,

Here a wreath, there a duct,

Which makes us ask,

So why a duct?

With a skew-skew here and a field-field there.

With an Ore here and Domain there,

With para here and free-free there,

$\mathrm{P}$ to the second power equals $\mathrm{B}$.

With a nil-nil here and potent there,

Reprise

And a Marcos here and a Gretchen there,

And a Kati here and a Peggy there,

And Delaram and Anthony,

Marianna, Shu, and Niu,

I like this group at any price,

For this group is very nice [don't be fooled that's a technical term!]

Here a wreath, there a duct,

With a skew-skew here and a field-field there.

With an Ore here and Domain there,

With para here and free-free there,

$\mathrm{B}+\mathrm{A}=\mathrm{P}+[\mathrm{B} \mathrm{B} \mathrm{B}]$

With a nil-nil here and potent there,

Reprise

\section{Ilya Kapovich}

The 4 years (1992-1996) that I was a student of Gilbert Baumslag at the CUNY Graduate Center, formed and defined me as a mathematician. Gilbert played a crucial role in this process. I learned many invaluable lessons from Gilbert during that time: How to think about mathematics, how to write mathematics, how to present and talk about mathematics, how to teach mathematics, and ultimately, how to be a mathematician. 
Gilbert gave me the time and the freedom necessary to discover what kind of mathematics most agreed with my talents and inclinations, and helped me chose a project with the right mix of geometry, algebra and combinatorics. I also learned from Gilbert that every graduate student is different, and there is no single formula or standard for advising them. My most intense mathematical interactions with Gilbert occurred when I was working on my first several papers. The process of writing the first of these papers, on small cancellation theory, was particularly agonizing (as I thought at the time). I must have gone through a hundred drafts of just the Introduction. After giving each draft to Gilbert, I would get it back densely covered in red ink comments. Every time I thought to myself: Is this pain ever going to end? Gilbert would always smile and say: “Ilya, don’t worry, we will get there, it is getting better!” Looking at that paper now, I think that Gilbert should have made me through a hundred iterations more! He worked hard to break some of my bad writing habits and to replace them by good ones. He taught me the importance of mathematical discipline and clarity, of avoiding complicated notation, overly long (as he said, Tolstovian) sentences and paragraphs, of keeping the main arguments short, clear, and well articulated, of breaking long proofs into shorter lemmas and propositions, and so on. These lessons define my mathematical writing style to this day, and I have tried to impart them on my own students. Gilbert taught me to direct my writing toward graduate students and mathematicians just entering the field, and not worry too much about trying to impress the experts and the snobs. A crucial conversation, also from the time of writing that small cancellation theory paper, concerned how to decide which mathematics is important. I told Gilbert that I was concerned that in my 20-plus page paper the crucial argument boiled down to a single page with a couple of pictures. Gilbert said: "Ilya, don't worry!" He said that he would tell me a big mathematical secret, which was that in any paper, no matter how long, there is usually a single short place where a crucial argument occurs. If a paper has two such places, then the paper is uncommonly good, and if it has three then, as he put it, "the paper is Inventiones level work." I learned from Gilbert that at the end you have to trust your own intuition about what kind of mathematics is interesting and important, and not be afraid to pose your own questions and problems. While I was struggling with the Introduction for one of my papers, Gilbert showed me the paper that he was working on the time, called "Musings on Magnus." When I read his Introduction, I was blown away: Not only did he use the pronoun "I" twice in the opening sentence, the first word of that sentence was "I." At the time, I could not imagine doing something so bold and brave in a mathematical paper. I learned from Gilbert the importance of storytelling in writing and presenting mathematics, and in my own papers I have been trying to tell stories ever since then.

So far, I had given Gilbert seven "mathematical PhD grandchildren," with two more on the way. In part through them, Gilbert's mathematical legacy and his genius continue to live on. I am proud and honored to have been Gilbert Baumslag's student.

Ilya Kapovich, Professor, Hunter College of CUNY. 


\section{Delarm Kahrobaei}

I graduated in 2004 under direction of Distinguished Professor Baumslag, writing a thesis related to residual solvability. Afterwards, I went to the University of St. Andrews (UK) as an assistant professor. Then for 12 years I was at CUNY, where I got my Full Professorship in 2015, and became a faculty member at the PhD Program in Computer Science. I am currently the chair of cyber security at the University of York (UK) in the Department of Computer Science. I also hold an adjunct professorship position at NYU, Department of Computer Science.

Gilbert Baumslag really helped me to pursue my passion to be a mathematician and computer scientist. He gave me encouragement and support. He also helped me to become a better person.

He encouraged me to dream big, and some of my major accomplishments in part are due to him. He told me examples of successful women mathematicians such as Hanna Neumann, who proved great theorems while she was raising children.

He was great at fostering an environment that created opportunities for other people. For example, he organized many conferences and seminars at City College and the Graduate Center focused on many aspects of group theory and some in cryptography. At these conferences/seminars, I met my key collaborators such as Vladimir Shpilrain.

Following the model put forward by Gilbert, I have since organized numerous conferences including many which that were designed to encourage women mathematicians and computer scientists.

He also encouraged me to take on leadership roles. For instance, he inspired me to create and be a director of York Interdisciplinary Centre for Cyber Security (University of York, UK, www.cs.york.ac.uk/security).

He was a superb lecturer and mentor. The first conference talk I ever gave was at the Albany Group Theory Conference. He coached me for over a month. He said I am a mathematical descendent of Philip Hall and I must give beautiful lectures.

Scientifically. I learned a lot from his mentorship to pay attention to details and also his interests in the interdisciplinary fields between combinatorial algebra and cryptography. This made me define a new career path to be a better scholar.

He had many students, and learning from his mentorship skills, I have had 8 PhD students (8 academic grandchildren for him), whom all graduated with degrees in mathematics and computer science and have gotten prestigious positions both in academia and industry.

I will miss him very much.

Professor Delaram Kahrobaei, Chair of Cyber Security Department of Computer Science (CSE 039), University of York, U.K. Professor of Computer Science (Adjunct), New York University, U.S.A. Doctoral Faculty in the PhD Program in Computer Science (Adjunct) Graduate Center, The City University of New York, U.S.A. http://www-users. cs.york.ac.uk/ delaram/. 


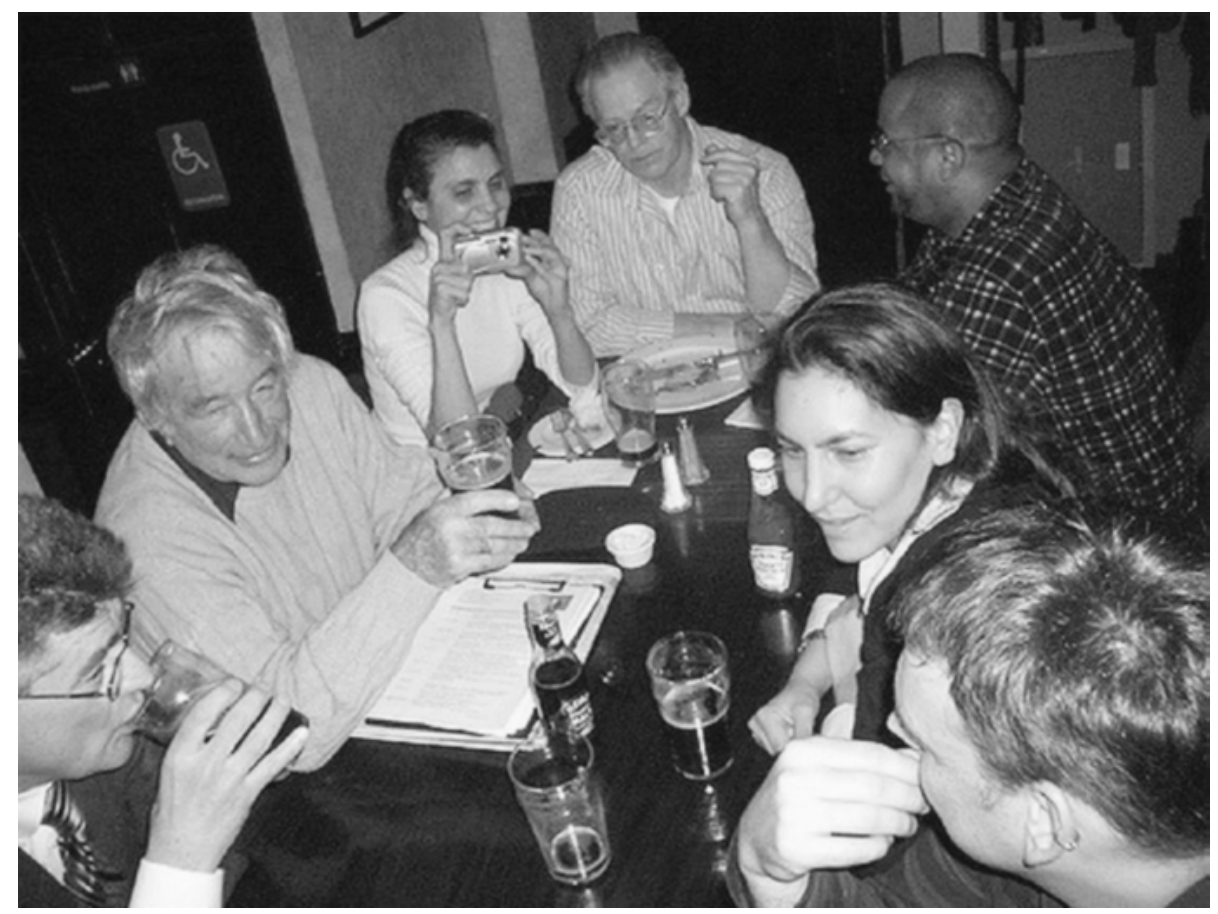

Taken on January 23, 2004, Dinner with Gilbert Baumslag after the NY Group Theory Seminar

Left to right: Bob Gilman, Gilbert Baumslag, Michael Thompson's girlfriend, Michael Thompson, Anthony E. Clement, Indira Chatterji and Murray Elder

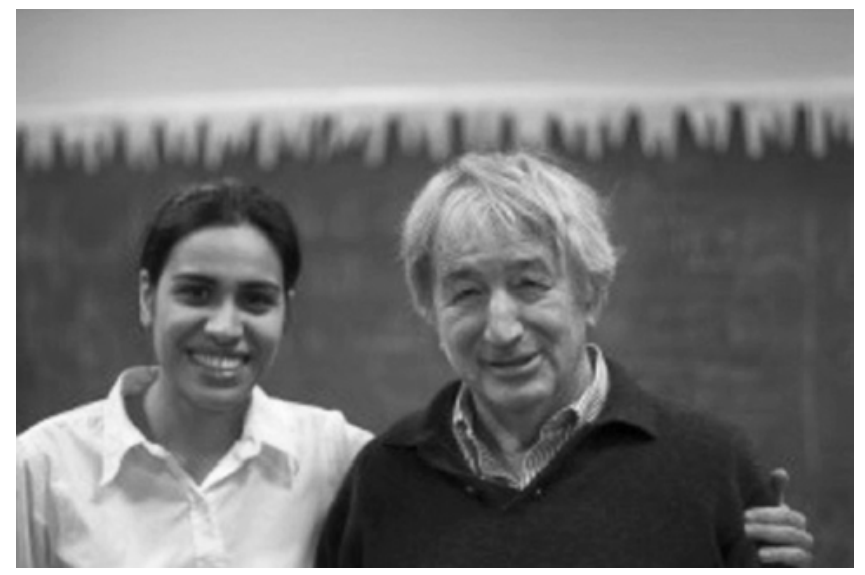

Delaram Kahrobaei and Gilbert Baumslag 


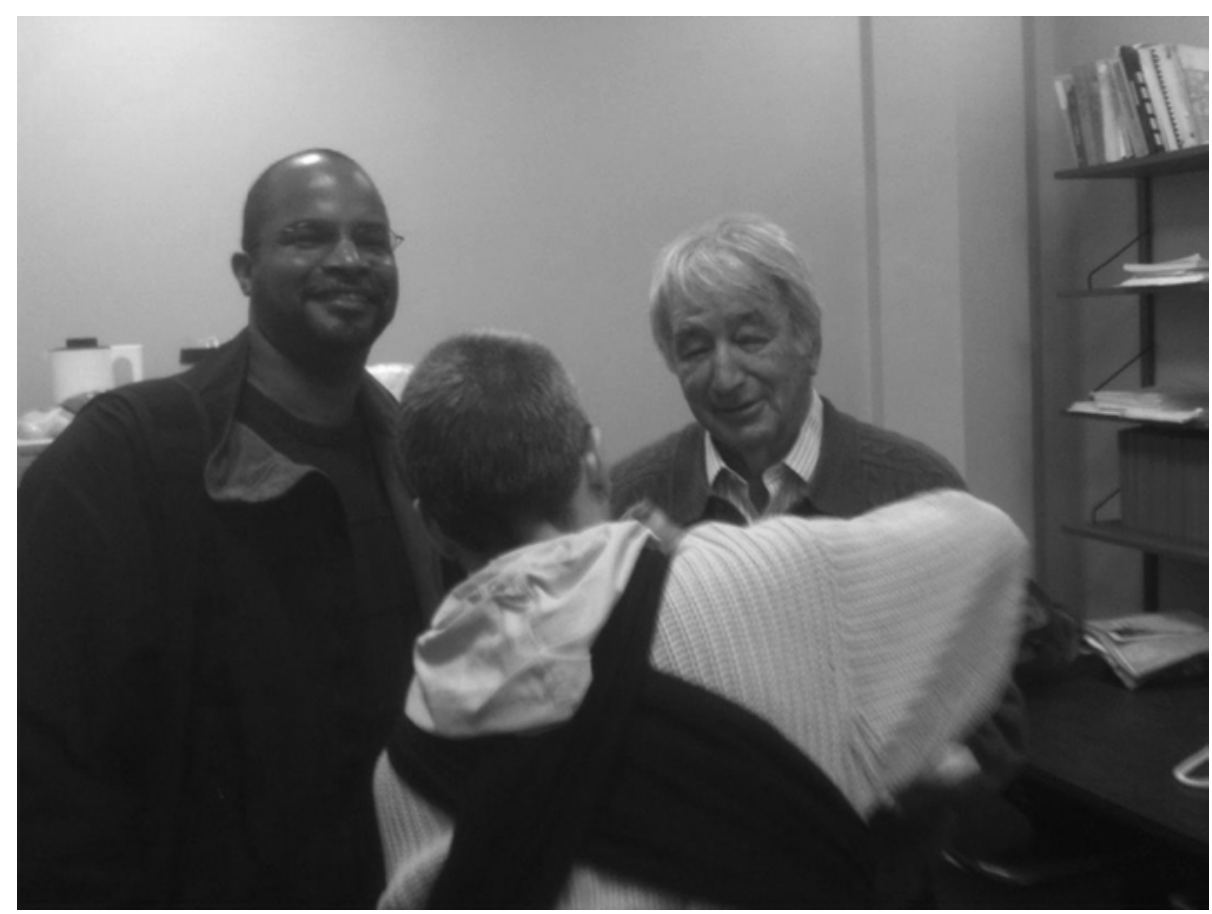

Taken on February 4th, 2011 at Gilbert Baumslag's office at the Graduate Center, NY Left to right: Anthony E. Clement (Gilbert's Student), Gretchen Ostheimer (collaborator) and Gilbert Baumslag

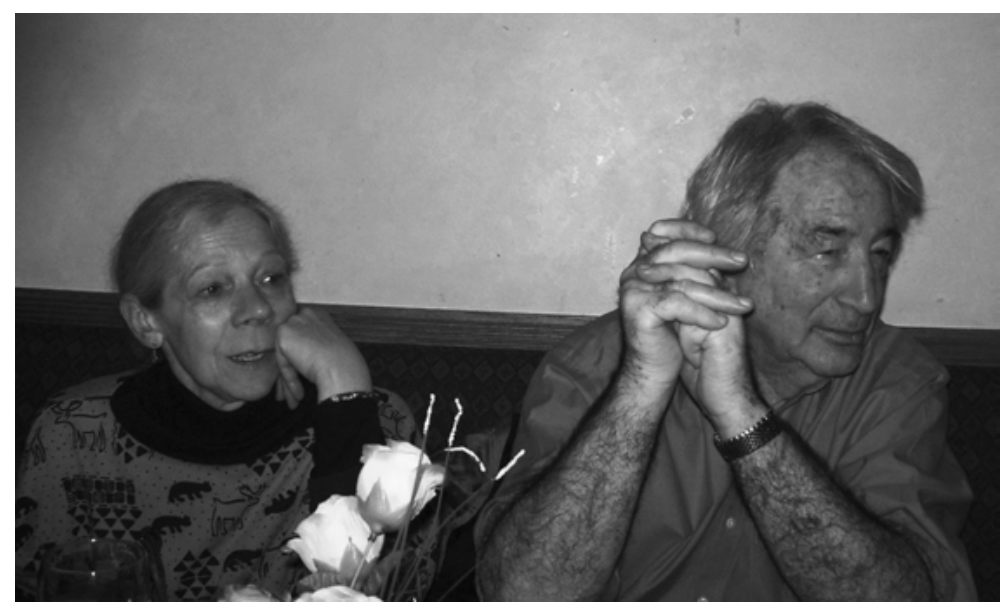

Kati Bencsath and Gilbert Baumslag 


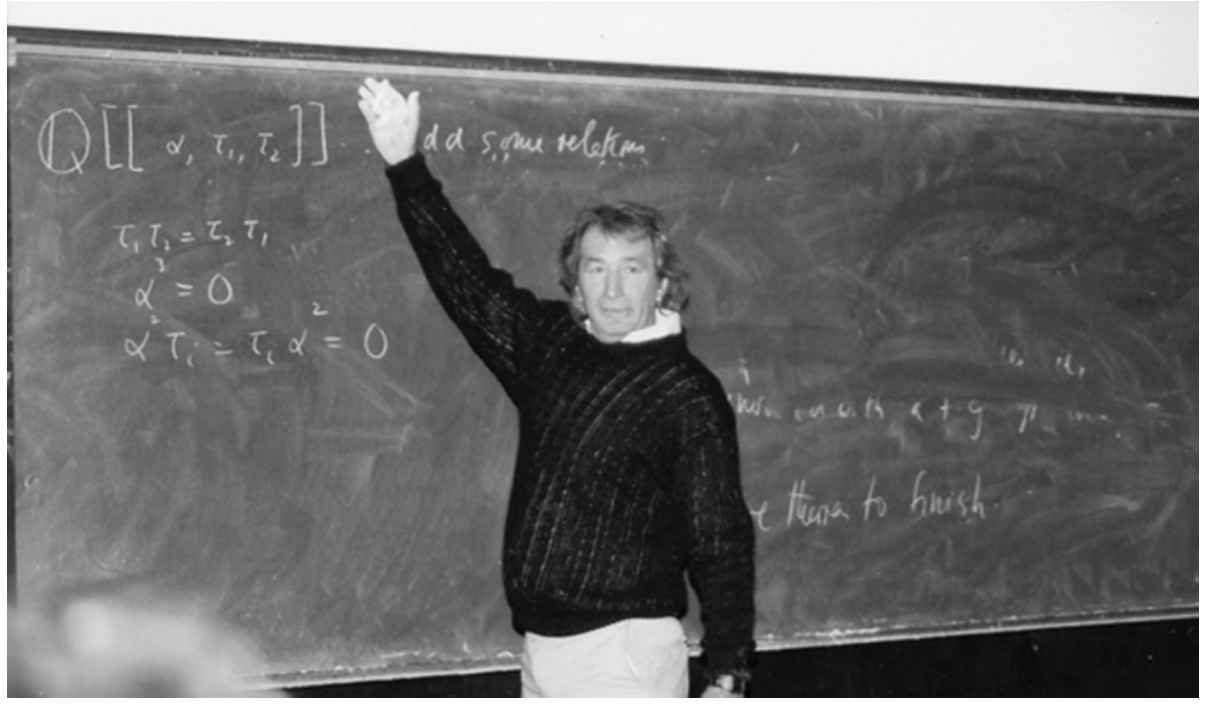

Young Gilbert Baumslag

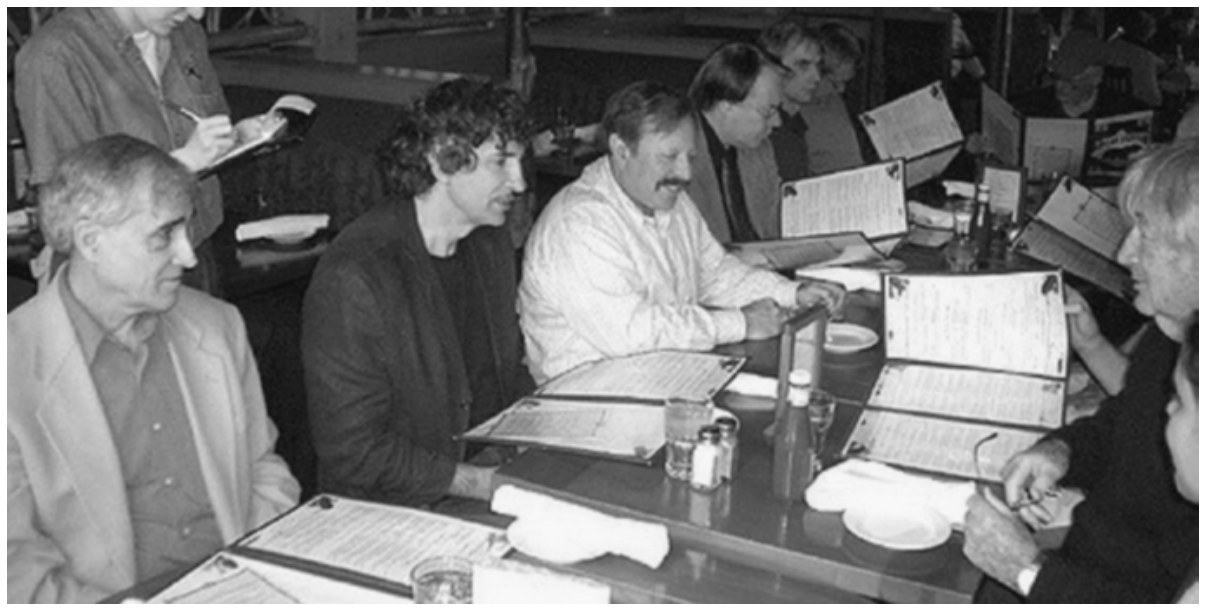

Left to right: Anthony Gaglione, Alexei Miasnikov, Ben Fine, Gerhard Rosenberger, guest speaker, Dennis Spellman, Gilbert Baumslag, and Delaram Kahrobaei 